\title{
Téoros
}

Revue de recherche en tourisme

\section{Ella Maillart, un « nouveau genre » de voyageuse (1923-1935)}

\section{Denis Voituret}

Volume 29, numéro 2, 2010

Tourisme et femmes

URI : https://id.erudit.org/iderudit/1024878ar

DOI : https://doi.org/10.7202/1024878ar

Aller au sommaire du numéro

Éditeur(s)

Université du Québec à Montréal

ISSN

0712-8657 (imprimé)

1923-2705 (numérique)

Découvrir la revue

Citer cet article

Voituret, D. (2010). Ella Maillart, un " nouveau genre " de voyageuse (1923-1935). Téoros, 29(2), 119-127. https://doi.org/10.7202/1024878ar
Résumé de l'article

Croisières et caravanes, ainsi s'intitule un ouvrage d'Ella Maillart, daté de 1950. Le titre résume les voyages exceptionnels effectués par l'écrivaine, photographe, journaliste, sportive et voyageuse de l'entre-deux-guerres. Les années 1920 voient, en compagnie de jeunes femmes sportives et érudites, la navigatrice émérite vivre pleinement puis renoncer, par la force des choses, à ses rêves de vie à bord d'un voilier. Durant les années 1930, après avoir effectué de premiers périples en solitaire en URSS, au Caucase, au Turkestan russe, la voyageuse se voit dans la prudente obligation de s'adjoindre un compagnon, Peter Fleming, afin de rejoindre ensemble et par leurs propres moyens l'Inde depuis Pékin. Le récit de cette expédition de forte notoriété, publié par chacun, fait date dans l'histoire des voyages. Nous pouvons observer, par les apparences, par les actes, par l'évolution des motivations de ses voyages, entre femmes puis parmi les hommes, et enfin par sa découverte d'elle-même, de quelle manière Ella Maillart, pionnière, se situe au féminin dans une histoire mixte des voyages précurseurs du tourisme moderne.
Ce document est protégé par la loi sur le droit d'auteur. L'utilisation des services d’Érudit (y compris la reproduction) est assujettie à sa politique d'utilisation que vous pouvez consulter en ligne.

https://apropos.erudit.org/fr/usagers/politique-dutilisation/ 


\title{
Ella Maillart, un «nouveau genre» de voyageuse (1923-1935)
}

\author{
Denis VOITURET \\ Docteur en histoire contemporaine \\ CRESOI, Université de La Réunion \\ de.voituret@wanadoo.fr
}

\begin{abstract}
RÉSUMÉ: Croisières et caravanes, ainsi s'intitule un ouvrage d'Ella Maillart, daté de 1950. Le titre résume les voyages exceptionnels effectués par l'écrivaine, photographe, journaliste, sportive et voyageuse de l'entre-deux-guerres. Les années 1920 voient, en compagnie de jeunes femmes sportives et érudites, la navigatrice émérite vivre pleinement puis renoncer, par la force des choses, à ses rêves de vie à bord d'un voilier. Durant les années 1930, après avoir effectué de premiers périples en solitaire en URSS, au Caucase, au Turkestan russe, la voyageuse se voit dans la prudente obligation de s'adjoindre un compagnon, Peter Fleming, afin de rejoindre ensemble et par leurs propres moyens I'Inde depuis Pékin. Le récit de cette expédition de forte notoriété, publié par chacun, fait date dans l'histoire des voyages. Nous pouvons observer, par les apparences, par les actes, par l'évolution des motivations de ses voyages, entre femmes puis parmi les hommes, et enfin par sa découverte d'elle-même, de quelle manière Ella Maillart, pionnière, se situe au féminin dans une histoire mixte des voyages précurseurs du tourisme moderne.
\end{abstract}

Mots-clés: Femmes, histoire, genre, voyage, représentations.

Les pionnières du tourisme, navigatrices, voyageuses, subissent dans les représentations médiatiques contemporaines soit un rappel immédiat de leur statut féminin, soit une comparaison avec le genre masculin, voire même parfois les deux simultanément.

Ilenvaainsidu tourismesportifmoderne.FlorenceArthaud, étonnante voyageuse (première femme à remporter en 1990 une course transatlantique en solitaire à la voile, la Route du rhum) hérite non seulement du surnom de "fiancée de l'Atlantique», mais encore d'attributs masculins : «Florence Arthaud, une femme qui en a!» (Métoudi, 1993 : 29). Il en va ainsi également pour le tourisme fondateur, méconnu, qui présente la particularité historique de s'écrire, in situ, au fur et à mesure du voyage et qui tente alors de réfléchir son image de lui-même, celle qu'il met en exergue volontairement, celle qu'il «donne à voir ».

Notre étude porte en effet sur les voyages de la sportive suisse, navigatrice, nomade, photographe "par goût», écrivaine et journaliste "par nécessité», Ella Maillart, «une femme du monde» (Morand, 1937) (voir le site des Amis d'Ella Maillart : www.ellemaillart.ch/index_fr.php).
Le travail d'histoire, une «connaissance par traces» (Prost, 1996 : 70), repose sur les sources primaires écrites souvent, témoignages oraux parfois, mais aussi sur l'iconographie, elle aussi source à part entière. Nous avons laissé de côté les reportages rédigés par Ella Maillart et sa correspondance privée. Nous nous sommes focalisé sur les ouvrages, récits dans lesquels l'auteure se confie à nous avec plus d'intimité que lors de ses articles pour la presse de reportage. Pudique, en réalité, Ella Maillart se livre très peu. Ainsi les récits de ses compagnes et compagnons directs de voyages complètent heureusement ce corpus. De même les photographies qu'ils nous ont transmises (reprises dans la littérature récente) donnent un éclairage précieux sur la personnalité de la voyageuse.

Les photographies et les récits transmettent en effet des représentations significatives de l'évolution radicale du système d'appréciation du voyage. À l'opposé de la vision masculine du récit de voyage qui ne permet pas d'appréhender réellement le "genre de la mobilité et les incidences du voyage dans la construction des identités sexuées» (Rogers et Thébaud, 2008 : 6), les écrits d'Ella Maillart, ses entretiens radiophoniques, les photographies permettent de révéler le caractère propre de 
l'image qu'elle donne d'elle-même et ainsi des rapports entretenus, dans la période de l'entre-deux-guerres, avec les autres, femmes et hommes, par la voyageuse d'un «nouveau genre».

L'insouciance présumée des années 1920, dites années folles, s'avère en réalité la conséquence directe du premier conflit mondial de 1914-1918. L'aspiration à la vie, à la liberté de la jeunesse sauve, femmes et hommes survivants, occasionne chez certaines et certains un rejet de la folie qu'ils prêtent aux sociétés européennes coupables de la Grande Guerre. Les modes de vie et de pensée de la "génération traumatisée» (Becker et Berstein, 1990 : 175) s'en trouvent profondément transformés; les aspirations de découverte d'autres mondes, d'autres sociétés en traduisent l'expression.

Le tourisme et le voyage ne font alors qu'un. Le tourisme de masse n'est pas inventé. La forme la plus simple de tourisme est en réalité une aventure. Le Tour britannique guide encore les systèmes de représentations. Dès le XVIII ${ }^{\mathrm{e}}$ siècle européen, la saison thermale aristocratique et l'amour de la campagne inventent les nouveaux terrains de jeux et les territoires des nouvelles sociabilités européennes (Boyer, 2002 : 394) et ultramarines (Voituret, 2008a). Le désir de rivages de la gentry, invention de la nouvelle harmonie du corps et de la mer, conjointement pénétré du spleen, du souci de l'environnement et de thérapeutique de l'esprit (Corbin, 1988 : 72) contribuent à asseoir l'image bénéfique et salvatrice du voyage.

Les voyageurs et voyageuses des années 1920 aspirent à un monde différent, à un tourisme novateur, porté par un autre idéal de vie que celui de la vieille Europe. Ils partent à la découverte d'autres sociétés et à la recherche d'eux-mêmes. Ils fuient l'élitisme du tourisme distinctif des groupes sociaux privilégiés. À l'échelle du XXe siècle, ils peuvent être comparés aux «routards» des années 1960-1970, souhaitant rompre avec la société de consommation des années «abusivement nommées Trente Glorieuses» selon Clément (2009 : 24). Par contre, la contribution des femmes au mouvement de 19201930, réaction philosophique, spirituelle à l'après Première Guerre mondiale est exceptionnelle. Leurs pratiques de voyages sont alors le fait de pionnières. La période de l'entre-deuxguerres peut être considérée comme un moment majeur où les femmes entrevoient les possibilités d'émancipation que leur offre à contrario la Grande Guerre. La fracture importante de la pyramide masculine des âges en Europe à la suite du conflit mondial de 1914-1918, auquel s'ajoute l'épidémie de grippe espagnole, a pour conséquence une diminution de la nuptialité et de la natalité. Le célibat et le rejet de la vie européenne projettent des hommes, de rares femmes, jeunes, sur la route des voyages bouleversant alors les espaces touristiques et les territoires de vies. En effet, les hommes survivants, pour certains, ne voient plus leur avenir en Europe et influencent la jeunesse des années 1920, pas aussi «folles» que des clichés historiques le laissent supposer depuis lors. Les années de véritable folie ne sont-elles pas en réalité celles de la guerre?

\section{Entre femmes, la liberté Insouciance sportive}

Ella Maillart fonde initialement sa vie sur les activités sportives et la lecture, et aborde alors ses rapports aux autres sous cette double appréhension du monde. Elle pratique dès l'enfance, de manière assidue, le ski avec sa mère l'hiver, et la navigation à la voile sur le lac de Genève au bord duquel, depuis l'âge de 10 ans, elle passe les six mois de la belle saison en famille au Creux de Genthod.

Là débute, selon Nicolas Bouvier (2002 : 5), «l'apprentissage de l'énergie» de la jeune femme qui reçoit une éducation moderne. "Sa mère, une Danoise indépendante et sportive, l'emmène chaque dimanche en montagne sur ces skis qui sont encore une excentricité d'Anglais. Son père est un commerçant genevois libéral dans ses façons, très informé du monde et qui lui en parle volontiers», souligne Bouvier (2002 : 5). Sa voisine et amie, Hermine de Saussure, surnommée Miette, l'initie à barrer un voilier, ce dont le frère aîné d'Ella Maillart «ne se souciait aucunement» (Maillart, 1993 : 14). De cette période, Ella Maillart (1993 : 14) écrit :

Le père de Miette lui apprit la manœuvre de son canot gréé en chasse-marée et à son tour elle me l'enseigna. C'était pour moi une aubaine, car mon frère ne se souciait aucunement de prendre sur son yacht une gamine de mon âge. Il appartenait à l'équipage d'un huit mètre cinquante de course, et nos repas du soir étaient animés par nos discussions sur les chances des compétiteurs.

Hermine de Saussure, des deux amies celle qui a la fortune suffisante, achète les voiliers successifs sur lesquels naviguent les deux amies en Méditerranée puis en Atlantique de 1923 à 1926 : Perlette, cotre de sept mètres vingt, Bonita, yawl de 14 tonnes, et le pilote du Havre transformé en yawl, Atalante.

La carrière sportive d'Ella Maillart est bien connue : elle crée à l'âge de 16 ans le Champel Hockey Club de Genève, un club féminin de Hockey sur gazon, est membre fondatrice du Ski Club féminin de Suisse en 1929, s'entraîne quatre mois de l'année dans les années 1930 et participe aux championnats du monde de 1931, 1932, 1933, 1934 (Girardin, 2003 : 9). Lors des Jeux olympiques de Paris de 1924, elle représente la Suisse en dériveur solitaire, unique concurrente féminine des épreuves de yachting (Voituret, 2008b : 700).

Les femmes sportives apparaissent en effet de manière manifeste sur la scène sociale de l'entre-deux-guerres. Les Jeux olympiques féminins, rebaptisés Jeux mondiaux féminins, sont contemporains de l'activité sportive de haut niveau d'Ella Maillart. Ils ont eu lieu à Paris en 1922, à Göteborg en 1926, à Prague en 1934 et à Londres en 1934 (Rosol, 2008 : 321). Pour Ella Maillart, la légitimité de ses pratiques sportives ne fait aucun doute. L'exclusivité masculine, élitiste, n'effleure pas la voyageuse. Son énergie et ses capacités lui permettent de ne renoncer à aucun de ses projets, fussent-ils jusqu'alors réservés aux seuls hommes.

\section{Croisière en Méditerranée, "voyage universitaire »}

Durant l'été 1925, la croisière de la Bonita a pour prétexte officiel de rallier la Grèce pour se rendre en Crète où l'équipage doit participer à une nouvelle campagne de fouilles dirigée par Marthe Oulié sur le site mis en valeur par l'École française d'Athènes (Vibart, 2004 : 10). Hermine de Saussure, 25 ans, et Marthe Oulié, 24 ans, toutes deux étudiantes à la Sorbonne et à l'École du Louvre inscrivent le voyage sous le signe de 
l'hellénisme et de l'archéologie. Ella Maillart, par son expertise acquise en 1924-1925, régatière aux Jeux olympiques de Paris, matelot professionnel en Angleterre, assure, selon ses dires, les fonctions de «seconde» à bord. En réalité Ella Maillart, indéniablement, mène l'embarcation, dans les tâches aussi bien délicates que difficiles : «Une demi-lune éclaire faiblement la jetée. Pas de brise. Bonita, remorquée par Ella du youyou, sort lentement, en même temps que quatre autres «lesteurs» » (Oulié, 2004 : 90). Devant les problèmes rencontrés lors de la navigation, la voyageuse fait preuve d'un véritable savoir de technicien. On lit ainsi dans les mémoires de Marthe Oulié : «Malgré le travail de la pompe, au début, le fond de la cabine demeure plein d'eau : la crapaudine marche mal. Ella, au prix de grands efforts, heurtée à tout instant des épaules et de la tête par le roulis doit soulever le linoléum, le plancher, et débarrasser avec la main la crapaudine des déchets qui l'encombrent» (Oulié, 2004 : 93). Plus loin, un autre passage évoque la compétence d'Ella Maillart, calme dans la tempête : «Au milieu de la nuit, la voix très calme d'Ella nous appelle : «Tout le monde sur le pont!» Le pic vient de casser près de la fourche! C'est grave, la grand-voile va être hors d'usage. Il faut amener et le vent fait rage» (Oulié, $2004: 115)$.

Nous empruntons à Loukia Efthymiou (2008 : 133-144) l'expression de «voyage universitaire». En effet, la croisière à bord de Bonita des cinq jeunes filles, navigatrices puis archéologues, a pour but de les mener en Grèce d'où elles rejoindront les fouilles françaises de Mallia en Crète (Oulié, 2004 : 261). S'ensuivront pour Marthe Oulié, chef de la "mission Oulié», plusieurs conférences, articles scientifiques et de vulgarisation. En 1934, Hermine de Saussure, épouse du directeur du Service des Antiquités, reçoit à Beyrouth la romancière Agatha Christie, en campagne archéologique avec son époux : " $[V]$ isite au service des Antiquités; délicieux déjeuner en compagnie de monsieur Seyrig, le directeur, et de son adorable épouse» (Christie Mallowan, 2006 : 45). En 1939, Ella Maillart, riche alors de ses nombreux voyages terrestres et récits appréciés par le monde, décide de devenir ethnographe cinéaste (Maillart, 2009a : piste 13), projet inabouti pour cause de Seconde Guerre mondiale.

Hellénistes, les jeunes femmes le sont par leurs études, par leurs lectures et par l'époque. L'antique pénètre alors de même les loisirs corporels. L'entre-deux-guerres voit en effet le développement du modernisme sportif qui présente deux tendances complémentaires : les sports anglais, impulsés par Pierre de Coubertin, afin de «conjuguer une tradition antique perdue et la modernité sportive» (Clastres, 2003 : 18), et les gymnastiques, inventées, entre autres par un officier de marine, Georges Hébert, créateur d'une gymnastique naturelle masculine aussi bien que féminine (Hébert, 1919 et 1912).

\section{Palestra, pantalons, casquettes et tabagie sportive}

Georges Hébert, de la Marine française, dès 1919, développe une gymnastique "naturiste», issue de sa Méthode naturelle pour femmes. Premier auteur à envisager «les faiblesses physiques des filles et des femmes comme le résultat d'un processus culturel interdisant tout entraînement » (Bazoge, 2006 : 200), il propose des exercices que peuvent pratiquer à égalité femmes et hommes. Les jeunes navigatrices de la croisière de
Bonita arborent la palestra, la tunique de gymnastique que Georges Hébert encourage à porter pour la pratique de sa méthode d'éducation physique moderne de plein air. Il crée en effet, en 1919, la Palestra de Deauville, un établissement pour les femmes inspiré de l'Antiquité grecque (Dumas, 1994). Le vêtement est adapté au contexte de la croisière et dénote une cohérence parfaite des sources de motivation de l'équipage entre Antiquité, philosophie naturelle et éducation physique hébertiste (Vibart, 2004 : 13), concordances d'intérêts profonds tendant à fonder leurs personnalités et à écrire les orientations de leurs itinéraires de vie.

La photographie de première de couverture de l'ouvrage de Marthe Oulié témoigne du port de la palestra par les trois jeunes femmes, Marthe Oulié, Hermine de Saussure et Ella Maillart. Plusieurs photographies de Mariel Jean-Brunhes, cinquième navigatrice, photographe de la fin de la croisière, confirment au sein de l'ouvrage le port de la tunique, ainsi que Marthe Oulié le note elle-même : «La journée se termine sur une expédition vers le fond du golfe de tout l'équipage, en maillot de bain et tunique de gymnastique, entassé dans le youyou» (Oulié, $2004: 134$ ).

Par contre lors des navigations en Atlantique, le climat breton impose le port de vêtements appropriés, masculins par la force des choses, comme le prouve, en 1926, la campagne de pêche de l'Atalante au large de l'île de Groix, préparatoire au grand départ des jeunes femmes vers les mers du Sud : «Il faut voir notre mine : la «capitaine», casquette groisillonne sur le nez, vareuse rouge et immenses bottes de caoutchouc [...]; le «second» en gros pantalon de ciré jaune et béret rouge» (Oulié, $2004:$ 184). «Comme la loi défend de vendre le poisson pêché sans rôle de pêche, et qu'on a pas de rôle de pêche sans être inscrit maritime, et que depuis Colbert, [sic] l'inscription maritime exclut les femmes, nous abandonnons le fruit de notre pêche - près de six cents francs - au bureau de bienfaisance de Groix» (Oulié, $2004: 189$ ).

Les habits masculins, pantalons, maillots, casquettes de marins, constituent non seulement l'équipement ad hoc, mais aussi permettent le déguisement, le camouflage comme lors du passage du Canal de Corinthe, selon le récit qu'en fait Marthe Oulié (2004 : 150) :

Il y a là une cabane des gardiens du canal [...] Il ne s'agit pas de montrer à ces gens, sans doute rébarbatifs, un équipage de cinq, dont deux ne sont pas majeurs, et dont le «capitaine» est une jeune fille... Mariel a endossé comme nous le tricot des marins à raies blanches et bleues, le pantalon et la casquette bleus. Nous prenons nos airs les plus virils, $[\ldots]$ et la casquette sur le nez, engoncée dans une grosse veste, d'une voix décidée, la «capitaine» va entamer les pourparlers [...] Ils avaient l'air plutôt étonné qu'ont ait envoyé le mousse pour discuter, nous avoue-t-elle en rentrant. Ils ont dit qu'ils nous laisseraient passer sans remorqueur si nous étions en nombre suffisant. Combien d'hommes êtesvous à bord ? ont-ils demandé... et j'ai répondu : cinq! Le port des vêtements de marins exprime également l'anticonformisme dont veulent faire preuve les jeunes femmes dans la bonne société de villégiature française lors de mondanités obligées en 1929 : 
La dernière traversée du Firecrest est pour se rendre à l'inauguration du nouveau golf de Deauville. Préparé à périr d'ennui, Alain [Gerbault] y retrouve, avec une joie folle, Hermine de Saussure et Ella Maillart qu'il impose au banquet prévu pour trois cents personnes. À l'heure dite, Hermine et Ella font une arrivée remarquée, sans chapeaux, jambes nues, jupes et vareuses à col marin (Vibart, 2001 : 177).

En effet, au-delà des notions de confort et d'apparence soumise à la mode, le vêtement n'est pas un objet neutre. Son histoire s'écrit en termes de construction sociale et d'assignation de genre. Cette histoire sociale reflète un système de normes et de définition des genres diversement construit dans l'espace et le temps. Ce qui est valable pour la vieille Europe ne l'est pas pour l'Océanie. Ella Maillart revendique à ce propos une indépendance et une indifférenciation manifeste, voire une confusion entre les sexes, car elle n'envisage nulle entrave à sa liberté de mouvement. La voyageuse représente, à son insu parfois, une figure majeure de l'évolution du masculin et du féminin. Si le genre est le sexe social, si l'étudier consiste à observer les assignations socialement construites à partir de l'identité sexuelle, Ella Maillart trouble les usages de la construction des rapports sociaux hiérarchiques, de la division entre masculin et féminin.

Ella Maillart, entre autres habitudes, fume la pipe. Cette pratique propose une facette supplémentaire à l'image que donne d'elle-même la jeune femme. Les années d'entre-deuxguerres prêtent à la consommation tabagique deux expressions : l'une masculine, sportive, liée à la détente après l'effort, particulièrement la pipe; l'autre féminine, liée à l'émancipation, lieu commun photographique durant les années 1920 (Bard, $1998: 38$ ).

En 1924, Ella Maillart trompe ainsi sa solitude anglaise : «Pour me donner l'impression d'être un peu plus chez moi dans ma chambre glaciale, il m'arrivait de bourrer ma vieille pipe, celle sur laquelle j'avais maintes et maintes fois tiré lors de joyeuses soirées d'hiver dans les refuges de montagne de mon pays» (Maillart, $1991: 33$ ).

Fumer la pipe n'est pas réservé totalement à la gent masculine comme le montrent les photos de mode tel le document «tenues d'intérieur pour fumeuses» (Bard, 1998 : 140) de la revue Fantasio en 1922 sur lequel posent deux jeunes élégantes, l'une fumant une cigarette, l'autre une pipe:

À ma grande surprise, Puck m'avait un jour invitée à venir passer chez elle le week-end suivant, et à ma plus grande surprise encore m'avait demandé si je voulais bien lui prêter ma seconde pipe. Par la suite elle avait quasiment bivouaqué devant ma porte pour que je lui raconte mes souvenirs de voile. Elle m'écoutait, ravie de sentir dans le creux de sa main la tiédeur du fourneau, alors que j'en suis sûre la fumée du tabac lui donnait des haut-le-cœur, car je fumais alors du gris que m'avait donné à Porquerolles un inscrit maritime (Maillart, $1991: 36$ ).

Ainsi, Ella Maillart fume la pipe en montagne, en ville et en mer. La voyageuse n'affiche peut-être pas tant son goût pour le tabac consommé en plein air que sa liberté : "Je pris donc le premier quart [...] J'allumais une pipe (j'étais arrivée à pouvoir la fumer sans avoir mal au cœur) qui me tint compagnie pendant ces heures solitaires. Fière de notre petit bateau isolé sur la mer, je me sentais envahie par une calme exaltation» (Maillart, $1993: 41$ ).

Hors du temps, au sein d'un espace apparemment sans limites, la jeune femme a le sentiment qu'une nouvelle forme de voyage au féminin et une nouvelle vie peuvent être inventées.

À l'image de George Sand, pionnière du XIX siècle, fumeuse, femme libre, hardie voyageuse, Ella Maillart ne revendique pas directement dans ses écrits une émancipation féministe, elle la vit. Sans l'ombre d'un doute, elle n'œuvre pas sciemment à la réduction des inégalités hommes-femmes, elle insuffle à sa vie intensément, profondément l'égalité entre les êtres humains.

L'apparence vestimentaire, pourtant capitale pour les représentations de genre, signifie pour l'une et l'autre, une simple coïncidence entre nécessité pratique et confort personnel. Le vêtement de voyage est masculin. George Sand considère que la mode ne permet guère aux dames d'aller et venir aussi librement qu'un homme. Elle reprend alors une habitude de jeunesse lorsque, pour aller à la chasse ou courir les bois, elle s'habillait en garçon, redingote en gros drap gris, un pantalon, un gilet (Cosnier, 2004 : 36). Ella Maillart sur la photographie À la découverte du Turkestan soviétique, 1932 (Maillart et Bouvier, 1991 : 11) arbore des vêtements attestant du confort recherché en voyage, sans renoncer au tricot rayé de matelot, et offre alors plus l'image d'une nomade désargentée que d'une touriste fortunée.

Pour bien prendre la mesure de l'anticonformisme et de l'innovation du mode de vie d'Ella Maillart, nous pouvons comparer la distance, physique et mentale, qui la sépare, par exemple, de jeunes excursionnistes en Bretagne en 1937. Sur une photographie étudiée par Christine Bard, Un piquenique des jeunes de La Feuillée partis en autocar au bord de la mer, dans le Finistère Sud (1937), "on distingue, dans un même groupe d'âge, les femmes gardant le costume breton et celles qui ont adopté des vêtements «de ville»» (Bard, 2007, photo $15: 18$ ). Traditions et usages ne permettent encore que le port de la jupe, qu'elle soit costume breton traditionnel ou de ville.

Lorsqu'en 1935 au Tsaidam Ella Maillart est photographiée «lisant la Jeune Parque de Paul Valéry» (Maillart et Bouvier, 1991 : $4^{\mathrm{e}}$ de couverture), elle porte chemise ample, pantalon et fume la pipe. Lors du même voyage, une autre photographie montre la voyageuse assise, plumant une volaille (Maillart et Bouvier, 1991 : 13). En se référant au magistral travail de Marianne Wex sur le langage féminin et masculin du corps photographié, Ella Maillart, vêtue d'une chemise large, d'un pantalon, chaussée de bottes, adopte une posture masculine, car «la position des jambes et des pieds le plus fréquemment observée chez les hommes assis consiste à écarter les cuisses en dirigeant les pointes des pieds vers l'extérieur» (Wex, $1993: 15)$ alors que les femmes ont rarement les genoux un peu écartés, les pointes des pieds sont également rarement tournées vers l'extérieur (Wex, 1993 : 19). Ella Maillart prend la "pose assise tout en largeur, typiquement «masculine»» (Wex, $1993: 21$ ). 


\section{Virilis, virilia, vir, vira}

Outre la modernité de leur pratique, nous pouvons nous interroger sur la part de force, de courage à prendre en compte dans les navigations des jeunes femmes (plus particulièrement Hermine de Saussure et Ella Maillart, les véritables femmes de mer) et la définition à lui donner. L'adjectif latin virilis est à la fois masculin et féminin. Il signifie certes mâle, masculin, mais également fort, vigoureux, courageux (Gaffiot, 1934 : 1682 ), tout comme virilia du genre neutre peut vouloir dire actes de courage, virils. L'attribution de la vigueur et du courage au masculin se trouve ainsi face aux actes des navigatrices pour le moins à discuter ici, le genre de la virilité à réenvisager. L'origine attribuée à vir, l'homme, ne peut-elle pas être vira, la femme? En tout état de cause, Hermine de Saussure et Ella Maillart font preuve d'une efficacité et d'une compétence tout à fait viriles, d'un genre non pas neutre, mais mixte.

La construction de la personnalité des jeunes femmes, particulièrement Ella Maillart, de leur rapport à la féminité et à la masculinité s'avère particulièrement innovante et significative devant les systèmes d'appréciations ayant cours alors en Europe. L'appropriation des pratiques sportives autant que professionnelles prouve l'autonomie, la liberté de décision d'Ella Maillart. La libération du carcan des apparences sociales et de genre de la jeune femme indique combien elle se joue des assignations sexuées. Par sa vision mixte et égalitaire du monde dans lequel elle aspire à évoluer, par son activité incessante, elle ne prête aux hommes que peu de pouvoir sur sa vie sans s'émouvoir de déclencher la peur de la confusion des rôles, le trouble dans le genre.

L'envie du voyage au féminin, l'innovation culturelle et sociale, se voit complétée par une aspiration philosophique à un monde sans guerre par les lectures et le contact avec des hommes survivants de la Grande Guerre. De l'insouciance sportive, Ella Maillart en arrive à un sentiment de rejet de l'Europe.

En 1918 en effet, Ella Maillart lit un livre par jour (Maillart, 2009c : piste 3). En 1995, elle déclare encore, à l'âge de 92 ans, à Marion Van Renterghem (1995: 12) du journal Le Monde: «On était jeunes [...] cette guerre nous avait chamboulées. Je me suis dit : l'Europe est foutue.» La conjonction du sport émancipateur et de l'aspiration au voyage vers un ailleurs pacifique entraîne chez Ella Maillart un irrémédiable et novateur choix de vie.

\section{Parmi les hommes, l'émancipation}

Le rêve commun à Ella Maillart et Hermine de Saussure de partir vivre dans le Pacifique naît « parce que nous avions rencontré Alain Gerbault qui était également un révolté contre la guerre - il était sorti vivant de la guerre qui lui avait tué ses meilleurs amis[.] Alain Gerbault avait décidé de partir sur son Firecrest, partir en solitaire par la force des choses puisque ses amis étaient morts, de partir vers le Pacifique, le Pacifique sud» (Maillart, 2003b : piste 6).

Croiser les récits des auteurs éclaire leurs actes. Ella Maillart, de ses conversations avec Alain Gerbault, révèle la part de rejet de l'Europe dans la décision du navigateur solitaire. Cette rencontre s'avère déterminante pour Ella Maillart qui, au même titre qu'Hermine de Saussure, n'envisage la vie que sous cet angle : "Avec mon amie Miette de Saussure [...] nous nous sommes dit, on va faire comme lui, on va partir pour le Pacifique, on va s'entraîner, on est de bonnes navigatrices [...] Cette idée d'aller vers une région où les gens vivaient sans faire des guerres épouvantables était une possibilité de vivre une vie intelligente» (Maillart, 2003c : piste 7).

Alain Gerbault écrit lui-même en 1924, lors du récit de sa traversée de l'Atlantique (qu'il réalise d'avril à septembre 1923) : «Je savais que je ne pourrais jamais plus mener dans une cité une existence sédentaire. La guerre me fit sortir de la civilisation. Je n'aspirai plus à y retourner» (Gerbault, 1924 : 18).

Les navigatrices rencontrent Alain Gerbault au port de Nice début 1923, à qui elles sont présentées par Virginie Hériot à bord de sa nouvelle goélette, Ailée (Vibart, 2001 : 59). Les projets d'Alain Gerbault de navigation en solitaire sur Firecrest influencent alors irrémédiablement Ella Maillart et Hermine de Saussure qui sont les premières dans la confidence, avec Pierre Albarran et Virginie Hériot (Vibart, 2001 : 60). «Aviateur pendant la Grande Guerre[,] lui et trois de ses compagnons avaient décidé de gagner le Pacifique et d'abandonner pour toujours un continent où de telles guerres étaient possibles. Ses amis avaient été tués, mais il n'avait pas renoncé à son projet; il était résolu à partir seul» (Maillart, 1993 : 47).

L'été 1923, Ella Maillart et Hermine de Saussure s'entraînent à la navigation semi-hauturière en Méditerranée et mouillent à Porquerolles : «C'est là que nous rencontrâmes deux écrivains sans lesquels nous aurions pu oublier cette Europe d'après-guerre qui nous inspirait que de l'antipathie. $\mathrm{Au}$ retour des tranchées, ils s'étaient révoltés ouvertement contre notre «civilisation». Au cours de nos entretiens, ils exprimèrent clairement ce que nous n'avions que confusément senti jusque[-]là» (Maillart, 1993 : 40). Le plus vif désir de vivre en mer, partagé alors par les deux amies, se trouve justifié et conforté à leurs yeux par le souhait de partir loin d'une Europe traumatisée. La découverte de civilisations différentes, la recherche d'empathie avec des sociétés humaines pacifiques les motivent.

\section{Professionnellement, à l'égal des hommes}

La compétence d'Ella Maillart pour la navigation et toutes les tâches inhérentes à la préparation, à l'entretien des bateaux n'est plus à prouver. Son énergie intrigue, dérange, enthousiasme. Lors de la préparation d'Atalante en Bretagne, 1926, elle raconte :

Sur le quai, cent quatre[-]vingts gueuses de vingtdeux kilos attendaient que je les transporte à bord. Et comme il s'agissait du lest le plus pratique, je craignais fort que durant la nuit on ne vînt en chaparder quelques-unes, aussi ai-je travaillé d'arrache-pied pour les soustraire aux convoitises, ce qui m'a valu plus d'une remarque flatteuse de la part des promeneurs du dimanche. - Ça[,] c'est du sport! Beau boulot. La dame[,] elle a pas peur de s'y atteler. C'est pas les nôtres qu'on verrait faire ça (Maillart, $1991: 145)$.

Ce qui ne va pas sans occasionner l'apparition de quelques nuages dans les relations des navigatrices avec des capitaines de thoniers : 
Les promeneurs, eux, avaient bavardé tant et plus. Ils n'arrivaient pas bien à comprendre ce que nous allions faire de l'Atalante. Ça devait être un «yak» puisque ce serait manœuvré par des dames... Et cependant l'une des «dames» en salopette crasseuse, travaillait ferme, et le «yak» avait été gréé de deux tangons comme on n'en voit que sur les thoniers. Pour les braves gens, c'était là un mystère... On ne s'en va pas pêcher le thon sans un patron à bord! Alors? Certains capitaines en étaient venus à nous prendre en grippe, Miette et moi, leur épouse ne s'étant pas fait faute de les traiter de fainéants, et de leur déclarer que leur soi-disant travail de forçat n'était tout bien considéré qu'une occupation pour les demoiselles (Maillart, 1991 : 146).

Lorsqu'Ella Maillart rédige, afin d'embarquer sur Volunteer en Angleterre, son «épître» d'embauche à l'attention du propriétaire, son post-scriptum résume sa conception mixte, égalitaire, de l'activité professionnelle de matelot et montre ainsi sa détermination : «P.S. [ :] À la relecture de cette lettre, je découvre que rien n'y laisse à deviner que je suis une fille, mais je demeure persuadée que la chose en soi est sans importance, tant il est vrai que tous les marins se ressemblent et que je suis marin d'abord et avant tout» (Maillart, $1991: 41$ ).

Ella Maillart, engagée en 1924 comme cuisinière, est enrôlée finalement comme matelot sur une barge de la Tamise, Volunteer, selon ses souhaits les plus chers. Le capitaine Jack Benett raconte :

Et puis un jour, mon second que j'étais allé voir à Brightlingsea m'a fait cette réflexion : «Mande pardon, Monsieur, cette fille, c'est p'têt' pas un cuistot de première, mais alors comme matelot, elle se pose un peu là.» Nous avons appareillé à la fin de la semaine et de fait, nous n'étions pas sur l'eau depuis dix minutes que le jugement du quartier-maître m'est apparu dans toute sa pertinence (Maillart, $1991: 16$ ).

La navigatrice fait preuve de compétence, de détermination et d'enthousiasme dès lors qu'elle peut vivre en mer : «Je n'avais en tête qu'une ambition : devenir un vrai bourlingueur des mers» (Maillart, 1991 : 12). L'identité biologique, sexuelle, de la navigatrice n'amène aucune interrogation sur sa légitimité à exercer les activités qu' elle aborde, aucun doute à propos de sa place en tant que femme au milieu d'hommes dans des sphères de pratiques jusqu'alors réservées aux seuls hommes. Être femme ou homme est une chose en soi sans importance, dit-elle, face à la fonction et à la nécessité, ici sur les océans, là, plus tard, au cour des grandes étendues terrestres.

\section{De la nécessité de gagner sa vie : écrire pour voyager}

On perçoit alors combien Ella Maillart rompt avec la classe de loisir, la leisure class, à l'oisiveté ostentatoire, fondatrice de la «révolution touristique» (Boyer, 2002 : 394). La voyageuse inaugure les nouvelles formes de tourisme, symbolisées par les offices de tourisme naissants, en Ouzbékistan soviétique par exemple, comme le rappelle Sara Steinert Borella qui cite Turkestan Solo : "le président de la Société de Tourisme prolétarien [...] est transporté de joie à ma vue : - Vous êtes notre première touriste. Camarades! Une touriste : une touriste de Paris, de France! - Qu'est-ce que c'est que touriste? lui demande son secrétaire» (Steinert Borella, 2006 : 62). Innovatrice, pionnière, Ella Maillart l'est donc assurément et pourtant comme elle le déclare elle-même 50 ans plus tard, elle a alors le sentiment d'avoir effectué un des derniers voyages à l'ancienne, sans pour autant en préciser la raison : «Après coup seulement j'ai eu conscience d'avoir effectué là un des derniers voyages à l'ancienne mode et j'en étais très fière» (Maillart et Domain, 1989, dans Fleming, 1989 : 416). L'auteure fait selon nous allusion aux modes de déplacements traditionnels qu'elle adopte par la force des choses, les deux aventuriers-reporteurs accomplissent en effet leur périple à cheval et à pied, seuls, avec guides et chameaux souvent, en caravane parfois. Pourtant, nous pensons que la voyageuse invente bien ici la charnière entre le voyage moderne de l'entre-deux-guerres au féminin (comme journaliste, envoyée spéciale, photographe au même titre que les hommes) et les derniers voyages des pionnières du XIX ${ }^{\mathrm{e}}$ siècle et du début du $\mathrm{XX}^{\mathrm{e}}$ siècle, telles Alexandra David-Néel ou Jane Dieulafoy. Des différences notoires existent en effet : Ella Maillart ne voyage pas avec son mari comme Jane Dieulafoy et ne possède pas la fortune d'Alexandra David-Néel qu'un mari attentionné gère à distance. La jeune femme doit subvenir à ses besoins tout au long de ses différentes expéditions. Un reportage, un livre, permet le prochain voyage: "Je vivais avec des reportages [...] avec les livres qui me donnaient un peu d'argent je pouvais penser à voyager plus loin» (Maillart, 2009b : piste 9).

À la suite de son premier périple en solitaire en URSS en 1930, la voyageuse publie en 1932 son récit Parmi la jeunesse russe chez l'éditeur Fasquelle, éditeur d'Alain Gerbault qu'elle rencontre par l'intermédiaire du navigateur. De bonnes critiques saluent la parution de l'ouvrage telle La revue de Paris en juillet 1932. Des articles d'Ella Maillart paraissent régulièrement dans des revues comme La Revue de Paris, mars 1934, le Journal de Genève, décembre 1930. Déjà, à l'issue de la croisière de 1925 les jeunes femmes publient un article dans la revue $L a$ Géographie, bulletin de la Société de Géographie. Le deuxième ouvrage de la voyageuse Des monts Célestes aux sables rouges paru en 1934 connaît un certain succès. À l'égal des hommes, les reportages en contrées éloignées permettent à Ella Maillart de vivre en voyage. À l'écriture pourtant, Ella Maillart préfère la photographie. Elle signe ses premiers articles sportifs du nom de Maillart-Leica (Girardin, 2003 : 9).

$\mathrm{Si}$ les voyages maritimes d'Ella Maillart s'accordent au féminin pluriel, ses voyages terrestres et montagnards, lorsqu'elle tourne définitivement le dos à la mer, se conjuguent au singulier puis au masculin lorsqu'un compagnon de voyage fait route avec Ella Maillart dans le plus célèbre de ses voyages, d'une durée de sept mois, de Pékin à l'Inde en 1935. Peter Fleming, correspondant du Times, était «un auteur à la mode, riche et célèbre» (Maillart et Domain, 1989, dans Fleming, 1989 : 417). Ella Maillart est l'envoyée spéciale du Petit Parisien en Mandchourie. Tous deux sont sur le pied d'égalité, journalistes reporteurs. Plus qu'un homme et une femme, ce sont deux caractères forts, deux solitaires qui s'associent : «Le dernier livre de Kini était intitulé Turkestan Solo et le mien One's company», souligne Fleming (1989 : 40). Le choix de l'itinéraire par le Turkestan chinois se porte sur celui 
prévu par la voyageuse : «Il faut que je décide de vous accepter avec moi» précise Maillart (2009c : piste 7). Enfin, comme le définit Fleming (1989: 40), «[n] ous ressentions encore une aversion irraisonnée à l'idée de voyager ensemble, mais celle-ci n'existait pas à côté de notre désir guère plus justifié d'accomplir cette expédition d'une façon ou d'une autre. En dernier ressort - à contrecœur et plutôt méfiants - nous nous trouvâmes associés. C'était contraire à tous nos principes». Nicolas Bouvier, dans sa préface à l'édition de 1989 de l'ouvrage d'Ella Maillart, Oasis interdites, le confirme : «Tous deux sont de fortes têtes, accoutumés à se débrouiller seuls et qui renâclent par moment contre la dépendance réciproque dans laquelle leur association les a placés; mais le projet qu'ils ont formé a plus de chances d'aboutir à deux » (Bouvier, 1989, dans Maillart et Domain, 1989: 8).

Par contre, in fine, lorsqu'en mai 1936 Peter Fleming conclut l'avant-propos à son récit du voyage commun, News from Tartary, il remercie Ella Maillart en ces termes :

Enfin je tiens à remercier Kini Maillart [...] À supposer que je puisse trouver les mots qui dépeindraient fidèlement sa vaillance, son endurance, sa bonne humeur, son tact de tels commentaires au début d'une narration prosaïque et sans prétention apporteraient une note à la fois trop conventionnelle et trop pompeuse. Çà et là, au cours du récit, je n'ai pu retenir certains témoignages d'estime. Mais la plupart du temps, j'ai laissé au lecteur le soin de se former une opinion sur une jeune fille qui parcourt des centaines de kilomètres à travers une région où nulle femme blanche ne s'était encore aventurée. Je ne doute pas qu'il ne la trouve, comme je l'ai fait, intrépide voyageuse et camarade sans égale (Fleming, 1989: 18).

La différence entre les deux voyageurs n'est pas tant le sexe que la finalité du voyage et le rythme que chacun souhaite lui donner. À l'opposé de Peter Fleming, pressé de rejoindre l'Inde et de retrouver ses compatriotes britanniques, qui « râle tous les matins parce qu'il n'a pas son Times à lire [...] Alors que $j$ 'aurais voulu m'arrêter pour vivre avec les nomades et les comprendre, il ne pensait qu'à l'ouverture de la chasse aux perdrix en Écosse, la rater était à ses yeux une chose impensable!» (Maillart et Domain, 1989, dans Fleming, 1989 : 416), Ella Maillart souhaite prendre le temps de la découverte. De plus, contrairement à Peter Fleming qui voyage pour écrire, Ella Maillart doit écrire pour voyager.

\section{Écrire, photographier pour témoigner et comprendre}

L'Asie centrale devient l'Ailleurs d'Ella Maillart. Elle se détourne de ses rêves de navigation, et arpente les montagnes afin de se découvrir elle-même et de découvrir les autres. Comme le souligne Sara Steinert Borella (2006 : 24), Ella Maillart s'éloigne de sa Suisse natale, mais respirera sa vie durant l'air des montagnes. Les sommets que la voyageuse franchit ne sont pas la finalité d'un exploit, la mâle conquête de la montagne, mais bien plus portes d'accès à d'autres mondes, à d'autres gens : "Maillart, for her part, continually returns to the mountains [...] However, her use of imagery differs from these male models in two ways: first the female version of the mountain summit does not balance upon conquest [...] As with her compatriots, mountains do, however, shape Maillart's gaze, and in her case, open the door to other worlds.»

La voyageuse est ethnographe. Au Turkestan russe, elle découvre les Kirghizes, les Kazakhs, les Ouzbeks. Au plus près des autres, des hommes matelots ou charpentiers des chantiers navals des débuts, aux jeunes femmes Tangoute rencontrées en chemin, Ella Maillart, humaniste, dans sa dédicace de l'exemplaire offert à Nicolas Bouvier, déclare avoir vécu «un voyage où il ne se passe rien, mais ce rien me comblera toute ma vie» (Bouvier, 1989 : 9). Lors d'une interview radiophonique en Angleterre en 1952, citée par Sara Steinert Borella (2006 : 23), Ella Maillart se félicite à posteriori d'avoir voyagé en état de relative pauvreté : «So, I am very grateful that I have been poor because it obliged me to travel in a different way from other travellers, or journalists, or explorers [...] I couldn't speak the langages of all these nomads and mongols and Tibetans, but because you try to live as they live and you are in the tent with them and so on, you're forced to imbibe something of their ways and thoughts and reactions.» Elle ajoute encore : "Je voulais voir ce que les nomades pensaient de la vie» (Maillart, 2009b : piste 9).

L'écriture, mais aussi la photographie témoignent des espaces, des gens qu'Ella Maillart se réjouit de rencontrer. Le don de ses 17000 négatifs au Musée de L'Élysée de Lausanne témoigne qu'elle pratique la prise de vue, le portrait avec plus de plaisir que le travail d'écriture. Peter Fleming (1989 : 43) décrit ainsi la connaissance de la photographie de la voyageuse :

Nous avions tous des appareils photographiques Leica; en fait, elle en possédait deux. C'est une habile photographe, connaissant la différence entre Isochrome, Superpan, et autres termes mystérieux; elle expose d'ailleurs de temps en temps à Paris. Toutes les vues que j'ai publiées avec ce livre ont été prises par moi [...] mais celles de Kini étaient infiniment supérieures aux miennes. Nos Leica, il est vrai, furent à la hauteur de la tâche; avantage fort appréciable de notre point de vue, on pouvait les manier d'une seule main; une grande partie des clichés ayant été pris en selle, il était bien commode de pouvoir, d'une main, tenir son cheval, et de l'autre, mettre l'appareil au point.

Le $1^{\text {er }}$ septembre 1939, alors qu'Adolf Hitler envahit la Pologne, Ella Maillart se trouve à Kaboul, en compagnie d'Annemarie Schwarzenbach, au bout de La voie cruelle, et pense "que tout le drame va recommencer» (Maillart, 2009b : piste 9). Elle se rend en Inde et «pensant qu'il me serait impossible de voyager après la guerre, je décidai d'y rester puisque je m'y trouvais. Afin de me procurer de quoi vivre, je commençai à écrire Gypsy Afloat avec l'espoir que suffisamment de gens l'achèteraient» (Maillart, 1988 : 360). Ella Maillart réside en Inde durant les années de guerre, écrit, et suit des enseignements de philosophie indienne. "Je voulais apprendre à me connaitre [...] Au bout du voyage, vous vous retrouvez vousmême» (Maillart, 2009b : piste 9).

Les deux voyages d'Ella Maillart, extérieur et intérieur, s'achèvent à Chandolin en 1997, dans son chalet d'altitude des Alpes du Valais suisse, son camp de base, qu'elle acquiert dès 1945 à son retour d'Inde. Elle quitte par la suite régulièrement 
son refuge pour guider des voyages culturels vers l'Asie. Ella Maillart poursuit en effet son œeuvre d'invention du tourisme moderne contemporain.

\section{Conclusion}

Nous avons voulu montrer, au travers de l'image que l'écrivaine-voyageuse donne d'elle-même, par ses écrits, sources primaires subjectives, par les photographies filtrées par la vision des compagnes et compagnons de route, par leurs récits, la figure d'exception que représente cette femme, exploratrice pionnière, auteure de nombreuses «premières» féminines, comme l'on nomme les conquêtes de sommets, les découvertes de territoires, habituellement masculines. Ella Maillart invente un nouveau genre de la mobilité, de l'indépendance, car elle effectue des périples, réussit des expéditions que les hommes n'ont pas réalisées avant elle.

Une exception confirme la règle néanmoins lorsqu'André Citroën refuse sa participation à la Croisière Jaune de 1931 sous prétexte qu'elle est une femme. Ella Maillart se trouve là confrontée à sa condition féminine. En fait, cela stimule plutôt que ne décourage sa volonté et sa détermination à parcourir les espaces qu'elle souhaite explorer à sa guise. Ella Maillart, en définitive, est la première jeune femme à rapporter un témoignage de son expérience de vie parmi la jeunesse russe (Winston Churchill invitera la voyageuse à son retour afin d'entendre son récit) et à arpenter le Caucase russe, la première chroniqueuse sportive à devenir envoyée spéciale en Mandchourie, la première femme aventurière à parcourir seule le Turkestan soviétique et à traverser la Chine et l'Asie centrale du premier $\mathrm{XX}^{\mathrm{e}}$ siècle en duo, la première voyageuse à rejoindre Kaboul en compagnie d'une autre femme à bord d'une voiture Ford décapotable des années 1930. Ella Maillart participe ainsi pleinement à la construction moderne des identités sexuées égalitaires, aux mutations du regard et ainsi à la nouvelle définition des zones d'interférence, d'indifférenciation des sexes, qui profitent aux femmes dans leur libre appréhension du monde.

\section{Références}

Association Étonnants voyageurs (s.d.) «Étonnants Voyageurs», Association

Étonnants voyageurs, <http://www.etonnants-voyageurs.com>, consulté le 25 mars 2010.

Association Les Amis d'Ella Maillart (s.d.) «Ella Maillart», Association Les Amis d'Ella Maillart, <http://www.ellamaillart.ch/>, consulté le 12 avril 2010.

BARD, Christine (1998) Les Garçonnes, modes et fantasmes des Années folles, Paris : Flammarion. $160 \mathrm{p}$.

BARD, Christine (2007) «Les photographies de famille commentées : une source sur l'habillement dans les classes populaires", Apparence(s), $\mathrm{n}^{\circ} 1$, $<$ http://apparences.revues.org/index79.html>, consulté le 22 mars 2010.

BAZOGE, Natalia (2006) «La gymnastique d'entretien au XX' siècle : d'une valorisation de la masculinité hégémonique à l'expression d'un féminisme en action", CLIO, Histoire, femmes et sociétés, n 23, p. 197-208.

BECKER, Jean-Jacques et Serge BERSTEIN (1990) Victoire et frustrations, 1914-1929, Nouvelle histoire de la France contemporaine-12, Paris : éditions du Seuil. $459 \mathrm{p}$.

BOUVIER, Nicolas (1989) «Ella Maillart et la chine centrale», préface DANS Oasis interdites, Ella MAILLART, p. 7-9. Paris : Payot.
BOUVIER, Nicolas (2002) Ella Maillart/Nicolas Bouvier, témoins d'un monde disparu, Carouge-Genève : éditions Zoé. $46 \mathrm{p}$.

BOYER, Marc (2002) «Comment étudier le tourisme?», Ethnologie française, $\mathrm{n}^{\circ}$ 3, vol. 32, t. XXXVII, p. 393-404.

CHRISTIE MALLOWAN, Agatha (2006) La romancière et l'archéologue ( $2^{\mathrm{e} e ́ d .), ~ P a r i s ~: ~ P a y o t . ~} 318$ p.

CLASTRES, Patrick (2003) «La décennie leplaysienne de Pierre de Coubertin, de la réforme des lycées aux Jeux olympiques (1883-1896)», Les Études Sociales, $\mathrm{n}^{\circ}$ 137, p. 5-19.

CLÉMENT, Gilles (2009) Le salon des berces, Paris : Nil éditions. 206 p.

CLIO Histoire, femmes et sociétés (2008) «Voyageuses», nº 28.

CORBIN, Alain (1988) Le territoire du vide, l'occident et le désir du rivage (1750-1840), Paris : Aubier. 407 p.

DUMAS, Jean-Philippe (1994) Aux origines de la «méthode naturelle»: Georges Hébert et l'enseignement de l'éducation physique dans la Marine française : Colloque de Joinville à l'Olympisme (École interarmées des sports de Fontainebleau : 24-25 mars 1994), <http://www.stratisc.org/ RIHM_83_24.htm\#_ftn35>, consulté le 16 avril 2010.

EFTHYMIOU, Loukia (2008) «Récits de voyage — quatre enseignantes à la belle époque», CLIO, Histoire, femmes et sociétés, n² 28, p. 133-144.

FLEMING, Peter (1989) Courrier de Tartarie (2 $2^{\mathrm{e} e ́ d .), ~ P a r i s ~: ~ P h e ́ b u s ~ l i b r e t t o . ~}$ $423 \mathrm{p}$.

GAFFIOT, Félix (1934) Dictionnaire illustré latin-français, Paris : Hachette. $1719 \mathrm{p}$.

GERBAULT, Alain (1924) Seul, à travers l'Atlantique, Paris : Grasset. 228 p.

GIRARDIN, Daniel (2003) Ella Maillart sur les routes de l'Orient, Lausanne : Musée de l'Elysée, Musée Olympique, Actes Sud. 159 p.

HÉBERT, Georges (1912) L'Éducation physique, ou l'entraînement complet par la méthode naturelle, Paris : Vuibert. 85 p.

HÉBERT, Georges (1919) L'Éducation physique féminine. Muscle et beauté plastique, Paris : Vuibert. 178 p.

L'ILLUSTRATION (1919) 2e semestre, p. 173-178.

MAILLART, Ella (1932) Parmi la jeunesse russe, Paris : Fasquelle. 254 p.

MAILLART, Ella (1934) Des monts célestes aux sables rouges, Paris : Grasset. $300 \mathrm{p}$.

MAILlART, Ella $(1988,1991)$ La voie cruelle ( $2^{\mathrm{e}}$ éd.), Paris : Payot. 372 p.

MAILLART, Ella (1989) Oasis interdites ( $2^{e}$ éd.), préface de Nicolas Bouvier, Paris : Payot. 242 p.

MAILLART, Ella (1991) La vagabonde des mers (2éd.), Paris : Payot. 419 p.

MAILLART, Ella (1993) Croisières et caravanes (2 éd.), Paris : Payot. 319 p.

MAILLART, Ella (2003a) Cette réalité que j'ai pourchassée, Carouge : Zoé. $175 \mathrm{p}$.

MAILLART, Ella (2003b) «Vos choix en partage : Ella Maillart par Yvette Z'Graggen", entretien radiophonique Radio Suisse Romande, 6 juillet 1979, DANS Cette réalité que j’ai pourchassée, Disque compact, piste 6. Carouge : Zoé.

MAILLART, Ella (2003c) «Ella Maillart 1903, écrivain-journaliste : «La passion de l'Asie» par Blaise Evard», entretien radiophonique Radio Suisse Romande, 29 décembre 1986, DANS Cette réalité que j’ai pourchassée, Disque compact, piste 7. Carouge : Zoé.

MAILLART, Ella (2009a) «Au retour de l'Inde», interview d'Ella Maillart par Vico Rigassi, 28 octobre 1945, DANS Entretiens avec Ella Maillart, le monde - mon héritage, Disque compact, piste 13. TSR/RSR et livret, Carouge : Zoé.

MAILLART, Ella (2009b) «Subjectif, entretien avec Ella Maillart par Jacques Donzel», entretien radiophonique Radio Suisse Romande, Chandolin, 25 octobre 1983, DANS Entretiens avec Ella Maillart, le monde - mon héritage, Disque compact, piste 9. TSR/RSR et livret, Carouge : Zoé. 
MAILLART Ella (2009c) «Ella Maillart rencontre Nicolas Bouvier, entretien en public avec Jean-Philippe Rapp», Radio Suisse Romande, Plume en liberté, Zurich, 25 septembre 1994, DANS Entretiens avec Ella Maillart, le monde - mon héritage, Disque compact, pistes 3-7. TSR/RSR et livret, Carouge : Zoé.

MAILLART, Ella et Nicolas BOUVIER (1991) La Vie immédiate, Lausanne : Payot, 24 heures. 199 p.

MAILLART, Ella et Catherine DOMAIN (1989) «Conversation avec Ella Maillart», DANS Courrier de Tartarie, Peter FLEMING, p. 415420. Paris : Phébus libretto.

MÉTOUDI, Michèle (1993) «Les femmes dans l'héroïsme sportif», Esprit, $\mathrm{n}^{\circ} 19$, novembre, p. 29-48.

MORAND, Paul (1937) «Une femme du monde», La Suisse, 28 avril. OULIÉ, Marthe (2004) Cinq filles en Méditerranée - 1925 — «quand j'étais matelot» ( $2^{\mathrm{e} e}$ é.), Rennes : éditions Ouest-France. 288 p.

PROST, Antoine (1996) Douze leçons sur l'histoire, Paris : Seuil. 341 p. ROGERS, Rebecca et Françoise THÉBAUD (2008) «Éditorial», CLIO Histoire, femmes et sociétés, «Voyageuses», n 28, p. 5-16.

ROSOL, Nathalie (2008) «Faites vos jeux! Le temps d'un olympisme réservé aux femmes", chapitre 10 DANS Les paris des Jeux olympiques de 1924, sous la direction de Thierry TERRET, p. 321-339. Biarritz : Atlantica.
STEINERT BORELLA, Sara (2006) The Travel Narratives of Ella Maillart, (En)Gendering the Quest, New York : Peter Lang Publishing. 141 p. VAN RENTERGHEM, Marion (1995) «Ella Maillart femme du globe», Le Monde [des livres], $\mathrm{n}^{\circ}$ 15756, 22 septembre, $\mathrm{p}$. XII.

VIBART, Eric (2001) Alain Gerbault vie et voyages d'un dandy révolté des années folles, Paris : éditions Payot \& Rivages. 373 p.

VIBART, Eric (2004) «Introduction, rencontre avec des femmes remarquables », dans OULIÉ, Marthe, Cinq filles en Méditerranée — 1925 "quand j'étais matelot», p. 9-14. Rennes : éditions Ouest-France.

VOITURET, Denis (2008a) «Images des loisirs de plein air dans La Réunion coloniale : le genre des pratiques (1860-1940)». Thèse de doctorat en histoire contemporaine, sous la direction d'Evelyne COMBEAU-MARI, La Réunion : Université de La Réunion. 696 p.

VOITURET, Denis (2008b) «Le Yachting à voile aux Jeux de 1924 : un entresoi international, des enjeux provinciaux et l'exception féminine", chapitre 22 DANS Les paris des Jeux olympiques de 1924, sous la direction de Thierry TERRET, p. 683-707. Biarritz : Atlantica.

WEX, Marianne (1993) Langage féminin et masculin du corps, reflet de l'ordre patriarcal, Louvain-la-Neuve : Academia-Erasme. 354 p.

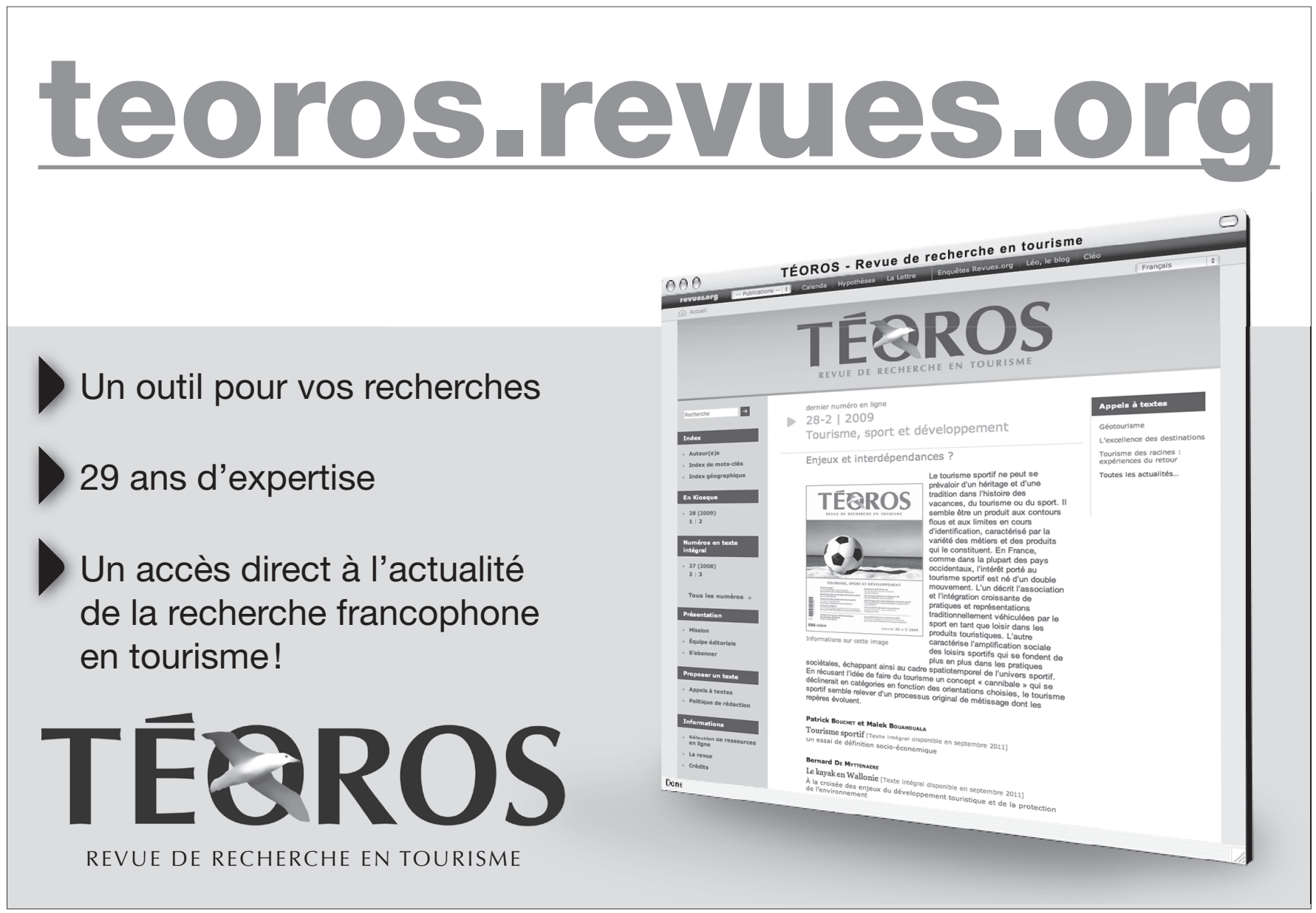

